\title{
WEIGHTED INEQUALITIES FOR THE GENERALIZED FOURIER TRANSFORM ASSOCIATED WITH THE CHEREDNIK OPERATOR ON THE REAL LINE
}

\author{
Najoua Barhoumi, RabiaA Ghabi and Maher Mili
}

\begin{abstract}
We consider the generalized Fourier transform associated with the Cherednik operator on $\mathbb{R}$ and we give the Hardy-Littelwood inequality and some weighted inequalities associated to this transform as particular case, we obtain the analogue of the Pitt's theorem.
\end{abstract}

Mathematics subject classification (2010): 42B10, 42A.

Keywords and phrases: Cherednik operator, generalized Fourier transform, Hardy-Littelwood inequality, weighted inequality, Pitt's theorem.

\section{REFERENCES}

[1] A. Achour AND K. TRimèche, La g-fonction de Littelwood-Paley associée à un opérateur différentiel singulier sur $(0, \infty)$, Ann. Inst. Fourier (Grenoble) 33, 4 (1983), 203-226

[2] J. J. BenEDETTO AND H. P. HeInIG, Weighted Fourier inequalities: new proofs and generalizations, J. Fourier Anal. and Appl. 9, 1 (2003), 1-37.

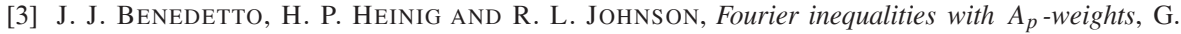
Ineq. 5, 5th Int. Conf., Oberwolfach/FRG 1986. Inter. Ser. Num. Math. 80 (1987), 217-232.

[4] S. Ben Farah and M. Mili, Pitt's theorem for the Dunk transform on $\mathbb{R}^{n}$, Math. Sci. Res. J. 12, 12 (2008), 291-301.

[5] M. Benjamin, Weighted norm inequalities for the Fourier transform, Trans. Amer. Math. Soc. 276, 2 (1983), 729-742.

[6] G. B. Folland, Real analysis: Modern techniques and their applications, J. W. and Sons INC, P. App. Math., New York, 2nd. ed. 1999.

[7] L. Gallardo, K. Trimèche, Positivity of the Jacobi-Cherednik intertwining operator and its dual, Adv. Pure Appl. Math. 1, 2 (2010), 163-194.

[8] G. H. Hardy and J. E. Littlewood, Some new properties of Fourier constants, Math. Ann. 97 (1927), 159-209.

[9] M. A. Mourou, Transmutation operators and Paley Wiener theorem associated with a Cherednik type operator on the real line, Anal. Appl. 8, 4 (2010), 387-408.

[10] H. R. PitT, Theorems on Fourier series and power series, Duke Math. J. 3, (1937), 747-755.

[11] E. STEIN AND G.Weiss, Introduction to Fourier analysis on euclidean spaces, Prin. Univ. Press 6th ed. 1990.

[12] K. TRIMÈche, The trigonometric Dunkl intertwining operator and its dual associated with the Cherednik operators and the Heckman-Opdam theory, Adv. Pure Appl. Math. 1, 3 (2010), 293-323.

[13] K. TRIMÈchE, Transmutation operators associated with a Cherednik type operator on the real line, Adv. Pure Appl. Math. 3, 4 (2012), 361-376. 